\title{
Clinico-microbial profile of Otomycosis in discharging otitis media in tertiary care hospital
}

\author{
Wagh Kailash B. ${ }^{1, *}$, Ghule Shubhangi B. ${ }^{2}$, Karande G. S. ${ }^{3}$, Mohite S. T. ${ }^{4}$ \\ ${ }^{1,2}$ Associate Professor, ${ }^{3}$ Professor \& Head, ${ }^{4}$ Professor \& Principal, Dept. of Microbiology, ${ }^{1,2}$ Dr. Ulhas Patil Medical College, \\ Jalgaon, Maharashtra, ${ }^{3,4}$ Krishna Institute of Medical Sciences, Deemed University, Karad, Maharashtra, India
}

*Corresponding Author:

Email: kwkailashwagh8@gmail.com

\begin{abstract}
Introduction: Discharging otitis media with superadded fungal infection is now become major otolarynological problems in India commonly seen in children and also in adults. The incidence has been increased in proportion to the wide spread use of topical antibiotics aural preparations.

Materials and Methods: Total 176 patients belonging to all age groups and both sexes(128 - male and 48 - female) were included. All were prediagnosed cases of discharging otitis media enrolled in the study.

Results: Out of 176 cases, 106 cases (76-male, 30-female) belong to acute suppurative otitis media (ASOM) and 70 cases (52male, 18-female) belong to chronic suppurative otitis media (CSOM). In mycological profile of both type of otitis media acute and chronic, 10 fungus isolated, 9 isolates were in chronic suppurative otitis media, of which 6 were of Aspergillus species and remaining 3 were belong to Candida isolates. Only one isolates of Candida albicans was seen in acute suppurative otitis media. Conclusion: We found that majority of the patients of chronic suppurative otitis media with superadded fungal infection as compared to acute suppurative otitis media.
\end{abstract}

Keywords: Acute suppurative otitis media(ASOM), chronic suppurative otitis media(CSOM), mycological profile.

\section{Introduction}

Otomycosis is frequently encountered in tropics. It also distributed world widely with higher prevalence in hot, humid and dusty areas.Superadded fungal infections of external auditory canal of patients of discharging otitis media are common now days. The incidence in such climate has increased in proportion to the wide spread use of topical antibiotic aural preparation. In Africa and other developing nations where disease prevalence could be as high as $11 \%$ with severe economic loss. ${ }^{1}$ In India it accounts to almost $3.5 \%$ of total patients attending outdoors. In past several years a worrying increase in the number of patients of having therapeutic failure has been reported along with increased frequency of recurrent and long lasting discharging otitis media. Frequent change of medical consultant, indiscriminate, haphazard use of antibiotics, half- treated, self treated along with itsrelated predisposing factors all lead to cause therapeutic failure hence there is chronicity in both (acute suppurative otitis media and chronic suppurative otitis media). This chronicity allow the growth of fungus, a variety of fungi, mostly the common saprophytes are the agents responsible for it.

In recent year prevalence of fungal infection in otorhinolaryngology is an increasing because of therapeutic failure cytotoxic chemotherapies and increased incidence of diabetes, immunosuppressive diseases and acquired immunodeficiency syndrome. Their evidence consistently proved by researchers in their study. Mittal A.et.al ${ }^{2}$ study showed mycotic otitis media in majority cases. Mohanty JC et.al ${ }^{3}$ showed more evidence of Aspergillus species along with bacteria in otomycosis. Recently Sampath CP et.al ${ }^{4}$ described predisposing factors and microbial picture of primary otomycosis. Most of the patients suffering from early otomycosis having complaint of severe itching which often progress to pain, hearing loss and often leading to tympanic membrane perforations. Various factors also have been proposed as predisposing factors for otomycosis including unhygienic mopping of ear, common cold, application of hot water/oil and more recently increased irrelevant use of topical antibiotics. Present study aims to find out clinico-microbial profile of otomycosis in otitis media.The objective of study is to find out the prevalence of fungal and bacterial pathogens and their association with different factors. (age, sex, predisposing factors)

\section{Materials and Methods}

The present study was a cross-sectional study. It was initiated following approval from the institutional ethics committee and written informed consent was obtained from all the study participants. The study was done by the Department of microbiology in collaboration with the Department of E.N.T O.P.D. Krishna Institute of Medical Sciences Deemed University, Karad. Patients who have been diagnosed to have ASOM and CSOM on the basis of clinical symptoms and signs were enrolled in the study. We identified the organisms including fungi and bacteria in both acute and chronic suppurative otitis media. The criteria to established superimposed otomycosis were based on following finding: 1) Presence of symptoms like itching,pain, deafness and otoscopy revealing masses of hyphae/ spores or a curd - like grey/white, 
blackish discharge 2) Demonstration of fungal elements in $10 \%$ potassium hydroxide preparation 3) Culture of clinical material on Sabourads Dextrose Agar. 4) Demographic details (age, sex), history associated with risk factors (unhygienic mopping, past history of antibiotic use/ear infections /surgery in the ear or head/ use of oil) collected from each study participant. Exclusion criteria were applied like patients not waiting for clinical examination and relevant investigation and also not adhering in treatment and follow up schedule.The external ear was cleaned with sterile wet cotton swab and type of discharge also observed. Auditory speculum was introduced in the ear and thin sterile cotton swab (manually made \& autoclaved) was passed through the ear and pus was collected from adjacent of the tympanic membrane and placed into sterile culture tubes. Three cotton swabs were used to collect the discharge and transported to laboratory for bacteriological and mycological examination. Routine investigations, serology for HIV and sugar for diabetes were done in all cases to rule out immunocompromised states. Processing: Out of collected three swabs, one swab was used for Gram staining and aerobic culture. For aerobic culture swab was processed on plain blood agar, MacConkey's agar and Chocolate agar and incubated aerobically at $37^{\circ} \mathrm{C}$ overnight. The second swab was processed for direct examination of fungal elements in $10 \%$ potassium hydroxide preparation and third swab was processed for fungal culture. Two sets of Sabouraud's dextrose agar with and without act idione were inoculated with the specimen, and incubated at $25^{\circ} \mathrm{C}$ and $37^{\circ} \mathrm{C}$ respectively. These were examined every day in the first week and then twice a week for the next three weeks for the rate of growth, colony morphology and pigmentation. Lactophenol cotton blue preparation, slide culture and germ tube test (in case of Candida albicans) were also performed to identify the species. A negative fungal culture report was given after 4 wks. Remaining procedure of their identification was done by standard procedures. ${ }^{5}$

\section{Observations and Results}

Demographic details (sex, age): Among 176 cases (128 were male and 48 female) 139 patients had unilateral discharge and 37 had bilateral discharge. Out of 176 cases, 106 cases (76-male, 30-female) belong to acute suppurative otitis media (ASOM) and 70 cases (52-male, 18-female) belong to chronic suppurative otitis media (CSOM). In mycological profile, 9 isolates were in CSOM and 1 fungal isolates was in ASOM.Descriptive statistics were used for representing the demographic details of the study participants. Age was categorized into five categories (in years) $(0-10$, 11-20, 21-30, 31-40 and above 40).

Table1: Age and sex distribution among acute suppurative otitis media.(ASOM)

\begin{tabular}{|l|c|c|c|}
\hline \multicolumn{1}{|c|}{ Age } & Total & Male & Female \\
\hline $0-10$ years & $35(32.02 \%)$ & $25(32.89 \%)$ & $10(33.33 \%)$ \\
\hline $11-20$ years & $31(29.25 \%)$ & $18(23.68 \%)$ & $13(43.33 \%)$ \\
\hline $21-30$ years & $27(25.47 \%)$ & $23(30.26 \%)$ & $4(13.33 \%)$ \\
\hline $31-40$ years & $13(12.26 \%)$ & $10(13.16 \%)$ & $3(10 \%)$ \\
\hline$>40$ & 0 & 0 & 0 \\
\hline Total & 106 & $76(100 \%)$ & $30(100 \%)$ \\
\hline
\end{tabular}

Table 1 shows that incidence of ASOM is more in male than in female.

Table 2: Age and sex distribution among chronic suppurative otitis media.(CSOM)

\begin{tabular}{|l|c|c|c|}
\hline \multicolumn{1}{|c|}{ Age } & No. & Male & Female \\
\hline $0-20$ years & $16(22.86 \%)$ & $12(23.08 \%)$ & $4(22.22 \%)$ \\
\hline $\begin{array}{l}21-40 \\
\text { years }\end{array}$ & $33(47.14 \%)$ & $24(46.15 \%)$ & $9(50 \%)$ \\
\hline$>40$ years & $21(30 \%)$ & $16(30.77 \%)$ & $5(27.78 \%)$ \\
\hline Total & $70(100 \%)$ & $52(100 \%)$ & $18(100 \%)$ \\
\hline
\end{tabular}

Table 2 shows that incidence of CSOM is more in male than in female.

Table 3: shows predisposing factors among all cases of otitis media

\begin{tabular}{|l|c|c|}
\hline \multicolumn{1}{|c|}{ Predisposing factor } & ASOM & CSOM \\
\hline $\begin{array}{l}\text { H/o Unhygienic mopping of ear } \\
\text { discharge / clean with sticks }\end{array}$ & $84(82.35 \%)$ & $22(31.42 \%)$ \\
\hline H/o application of hot water/ oil & $51(50 \%)$ & $12(17.14 \%)$ \\
\hline H/o common cold & $62(60.78 \%)$ & - \\
\hline H/o Topical antibiotic used & $22(21.57 \%)$ & $60(100 \%)$ \\
\hline H/o Allergic rhinitis & $3(2.94 \%)$ & $26(43.33 \%)$ \\
\hline
\end{tabular}




\begin{tabular}{|l|c|c|}
\hline H/o Tonsillitis & $12(11.76 \%)$ & $14(23.33 \%)$ \\
\hline $\begin{array}{l}\text { H/o Discharge following } \\
\text { U.R.T.I. }\end{array}$ & - & $16(26.67 \%)$ \\
\hline H/o Presence of chronic illness & $2(1.96 \%)$ & $4(6.67 \%)$ \\
\hline H/o otitis media in family & $4(3.92 \%)$ & - \\
\hline H/o faulty breast feeding & - & - \\
\hline H/o bottle feeding & $2(1.96 \%)$ & - \\
\hline H/o Smoking & - & $4(5.71 \%)$ \\
\hline H/o Chronic pharyngitis & - & $12(20 \%)$ \\
\hline H/o ear surgery & - & $3(5 \%)$ \\
\hline H/o exathematous fever & - & - \\
\hline
\end{tabular}

Table 3 shows that out of 106 cases of acute suppurative otitis media, H/o unhygienic mopping of ear cases $(82.35 \%)$ is found as a most common predisposing factor followed by $\mathrm{H} / \mathrm{o}$ common cold $(60.78 \%)$, H/o application of hot water/oil (50\%) and H/o irrelevant use of topical antibiotics (21.57\%). In 70 cases of chronic suppurative otitis media, H/o use of antibiotics (100\%) commonly seen followed by H/o allergic rhinitis (43.33\%), H/o unhygienic mopping of ear (31.42\%), H/o repeated ear discharge after upper respiratory tract infections (26.67\%) and $\mathrm{H} / \mathrm{o}$ tonsillitis $(23.33 \%)$.

Table 4: Incidence of fungal isolates among ASOM \& CSOM culture positive cases

\begin{tabular}{|l|c|c|c|c|}
\hline Organisms & ASOM & \% & CSOM & \% \\
\hline Candida albicans & 1 & $100 \%$ & 3 & $33.33 \%$ \\
\hline Aspergillus fumigatus & - & - & 5 & $55.56 \%$ \\
\hline Aspergillus flavus & - & - & 1 & $11.11 \%$ \\
\hline Total & 1 & $100 \%$ & 9 & $100 \%$ \\
\hline
\end{tabular}

Table 4 shows i) Majority of fungal isolates in CSOM than ASOM.

ii) In mycological profile of both otitis media, 9 isolates were in chronic suppurative otitis media, of which 6 (66.67\%) were of Aspergillus growth. Aspergillus fumigatus being the commonest isolates and remaining $3(33.33 \%)$ were Candida isolates. Only one fungal isolates of Candida albicans was present in acute suppurative otitis media.

\section{Discussion}

The results are compared with the study conducted by other researchers. Therapeutic failure and its associated factors are always responsible for chronicity in both acute suppurative otitis media and chronic suppurative otitis media this chronicity allow the growth of fungi. Acute suppurative otitis media is defined by convention as first 3 weeks of a process in which the middle ear shows the sign and symptoms of acute inflammation which can lead to further complication such as chronic suppurative otitis media developed from such previous acute inflammatory state. There is no accepted time divided between acute and chronic, according to some authorities this range is from 2 wks to 3 months. Fungi can either be the primary pathogen or be superimposed on bacterial infections or can be secondary pathogen in previously perforated tympanic membrane. ${ }^{6}$

Demographic details (sex, age, side of involvement)
We found that majority of the study participants belonged to either paediatric or adolescent age group. In such age group a shorter, wider and more horizontal location of eustachian tube is responsible for transmitting infection from nasopharynx to middle ear. Our study indicates that maximum number of cases of otomycosis occurred in the age group between 0 to 10 years and males are predominantly affected than females. (Male to female ratio is 2.5:1 in ASOM.). The incidence of fungal infection was comparatively less in the age group above 40 years. The high incidence of acute suppurative otitis media in pediatric age group and males was also found in studies carried out by other researchers Deshmukh CT.et.al ${ }^{7}$, D. Vijaya et al., ${ }^{8}$ De A. et al, ${ }^{9}$ and Kumar P et al. ${ }^{10}$

We found in chronic suppurative otitis media incidence was commonly found in age group of 21-40 years and males are predominantly affected than females.( male to female ratio is 2.8:1 in CSOM). Similar findings were reported by Fule RP.et. al, ${ }^{11}$ Kulkarni RD.et al, ${ }^{12}$ D Vijaya et al, ${ }^{8}$ Sinha A.et al, ${ }^{13}$ and Poorey VK.et al. ${ }^{14}$ The reason behind male predominance could not be found out. The incidence of otomycosis was found to be more in males as they work outdoor and hence more exposed to dust which may lead to deposition of fungal spores. In our study rural population (92) was more commonly affected than urban (70) population. Our findings are consistent with studies of Taneja MK.et al. ${ }^{15}$ 


\section{Clinical presentation}

In clinical spectrum of our study of ASOM, otalgia is the prominent symptom $(84.31 \%)$ followed by pulsatile discharge $(50.98 \%)$, febrileness $(47.06 \%)$, fullness of ear $(31.37 \%)$, deafness $(25.49 \%)$ and tonsillitis $(11.76 \%)$. In studies of De A, ${ }^{9}$ tonsillitis was the commonest sign and in studies of Taneja MK. et $\mathrm{al}^{15}$ deafness was the commonest presentation. In case of CSOM, reported symptoms were $(25.5 \%)$ deafness, $(31.4 \%)$ had blocking sensation in the ear, $(84.3 \%)$ had ear ache and $(51 \%)$ had pulsatile ear discharge.Other studies showed similar findings. Gulati J. et $\mathrm{al}^{16}$ reported otorrhea, deafness and pain as commonest findings. In our study out of 106 cases of acute suppurative otitis media, 79 cases had purulent discharge found in 79 cases $(77.45 \%)$, mucopurulent in $21(20.59 \%)$, mucoid in $4(3.77 \%)$ and. blood mixed in $2(1.96 \%)$. In cases of chronic suppurative otitis media, out of 70, mucopurulent discharge was found in 46 $(76.67 \%)$, purulent discharge in $9(8.57 \%)$, mucoid in 7 (10\%) and blackish discharge in $4(6.67 \%)$ cases. Blackish papery discharge in 4 cases $(6.67 \%)$ was typically seen in patients of diabetes mellitus which yields fungal growth on culture.

\section{Predisposing risk factors}

Out of 106 cases of acute suppurative otitis media, H/o unhygienic mopping of ear cases $(82.35 \%)$ is found as a most common predisposing factor followed by H/o common cold (60.78\%), H/o application of hot water/oil $(50 \%)$ and $\mathrm{H} / \mathrm{o}$ irrelevant use of topical antibiotics $(21.57 \%)$. In 70 cases of chronic suppurative otitis media, H/o use of antibiotics (100\%) commonly seen followed by H/o allergic rhinitis (43.33\%), H/o repeated ear discharge after upper respiratory tract infections (26.67\%) and H/o tonsillitis (23.33\%).Our data were generally comparable with these previous studies.Unhygienic mopping of the ear may transmit the infection from an external environment to the middle ear and is more commonly seen amongst the patients from rural areas. ${ }^{15}$ Prior intake of antibiotics could also have suppressed bacterial growth. This finding correlated with the study conducted by Mawson S.et.al ${ }^{17}$ and Kunelskaya et.al ${ }^{18}$ reported that in most of cases the fungal process in middle ear developed as a result of topically treated with antibiotics and steroids.

\section{Microbiological consideration}

Among 106 cases of ASOM, a total of 117 organisms have been grown in the culture of which 116 bacterial isolates and only one belong to fungal isolate sand it was Candida albicans. In 70 cases of CSOM, 9 fungal isolates were found. Among them 6 (66.67\%) were of Aspergillus growth. Aspergillus fumigatus being the commonest isolates and remaining 3 (33.33\%) were Candida isolates. Fungal organism being the etiological agent for acute suppurative otitis media has been rarely reported in the literature.This findings in is concurrence with the study of De A. et $\mathrm{al}^{9}$ and D Vijaya et al. ${ }^{8}$ Isolates of Aspergillus were more than Candida. Among the fungal etiology in chronic suppurative otitis media, most of studies showed commonly isolated organisms were Aspergillus species and Candida species. Earlier study from India, reported higher isolation rate of Aspergillus species (60.2\%) as compared to Candida species (17.6\%). This finding is in concurrence with our study in relation to chronic suppurative otitis media concerned.

Following all studieshaving Aspergillus fumigatus and Aspergillus spp.are predominat isolates. In Juyal D. et. $a^{19}$ study among the 107 cases, fungal isolates $83(77.57 \%)$ were isolated in chronic suppurative otitis media study. Of the 83 fungal culture positive cases, the predominantly isolated fungi were Aspergillus species (47\%), Candida species (41\%), and Penicillium species(9.6\%) among the Aspergillus, the predominant species were Aspergillus flavus (17 isolates) and Aspergillus niger (12 isolates) Candida albicans (19 isolates) and Candida tropicalis ( 9 isolates) were the commonly isolated species of Candida. In study of Jain SK and Agrawal $\mathrm{SC}^{20}$ Aspergillus niger $(56.3 \%)$ was the commonest isolate followed by Aspergillus fumigatus (15.6\%) Aspergillus flavus (4.7\%) and Candida albicans $(6.3 \%)$, Mucor(6.3\%) (4\%) Penicillium spp.(4.7\%) and other (3.1). In the study of BavejaCP. et. $\mathrm{al}^{21}$ (1993), Aspergillus fumigatus (52\%) was the commonest isolate followed by Aspergillus niger (28\%) and Candida albicans (12\%) Penicillium spp.(4\%) and fusarium (4\%). In study of Mittal A.et.al ${ }^{2} 42$ out of 103 Aspergillus flavus $20(47.62 \%)$ was the commonest isolate followed by Aspergillus niger 4(9.52\%) Aspergillus fumigatus 3(7.14\%), Aspergillus terreus 4(9.52\%), Aspergillus glauces 01(02.38\%) and 6 were belong to Candida albicans and 1 was belong to cladosporum. In the study of Mohanty JC.et $\mathrm{al}^{3}{ }^{3}$ Aspergillus niger $(41.1 \%)$ was the commonest isolate followed by Aspergillus flavus (24\%) and Candida albicans $(5.5 \%)$. Rajesh KY.et.al ${ }^{22}$ showed among the fungal etiology in chronic suppurative otitis media, in their study, Aspergillus species comprised of more than $45 \%$ of the total fungal isolates, whereas approximately $40 \%$ of the total microorganisms were species of Candida. Aspergillus flavus and Aspergillus niger were the most common Aspergillus species. Among the Candida species, the most common isolates were Candida albicans and Candida tropicalis. A recent study by Aneja KR.et.al ${ }^{23}$ from India reported Aspergillus in $86.8 \%$ of patients with Aspergillus niger $(39.8 \%)$ and Aspergillus flavus (16.6\%) to be the most common species. Shrestha BL. et al. ${ }^{24}$ among 230 isolates Aspergillus spp. 16 (6.9\%) Candida spp.8 (3.4\%).In Dhingra R.et al ${ }^{25}$ study out of total $36(100 \%)$ Aspergillus species 32(88.8\%) Aspergillus flavus 16(44.4\%) Aspergillus niger 16(44.4\%), Candida albicans $4(11.2 \%), \quad$ Candida tropicalis 
(0\%).In study of Sampath CP. et $\mathrm{al}^{4}$ Aspergilli were the most common isolates (80\%) followed by Penicillium (8\%), Candida albicans (4\%), Rhizopus $(1 \%)$, and Chrysosporium (1\%), the last being reported for the first time in otomycosis. Among Aspergilli, Aspergillus nigercomplex $(38 \%)$ was the most common followed by Aspergillus fumigates complex (27\%) and Aspergillus flavus complex (15\%).

Aspergillus was most common causative agent of otomycosis in majority of studies. But its species variation as reported from place to place is due to the geographical pattern of fungus at different places. The commonest fungus isolated was Aspergillus niger among the other Aspergillus species in most of studies. Ali $\mathrm{ZM}^{26}$ showed the most common fungal pathogens were Aspergillus niger ( 8 cases) followed by Aspergillus flavus(2 cases), Aspergillus fumigatus, PenicilliumSpp., Candida albicans, C. parapsilosis and Rhizopus Spp. each 1 case. Haneefa S.et.al ${ }^{27}$ showed of 173 cases of chronic suppurative otitis media, Aspergillus forms the major fungal pathogens $25(14.45 \%)$, followed by Candida $13(7.51 \%)$.

Table 5: various studies shows their commonly isolated fungus in otomycosis

\begin{tabular}{|c|c|}
\hline Fungi & $\begin{array}{l}\text { Name of author and year and commonly isolated } \\
\text { fungus in their study }\end{array}$ \\
\hline Aspergillus species: & 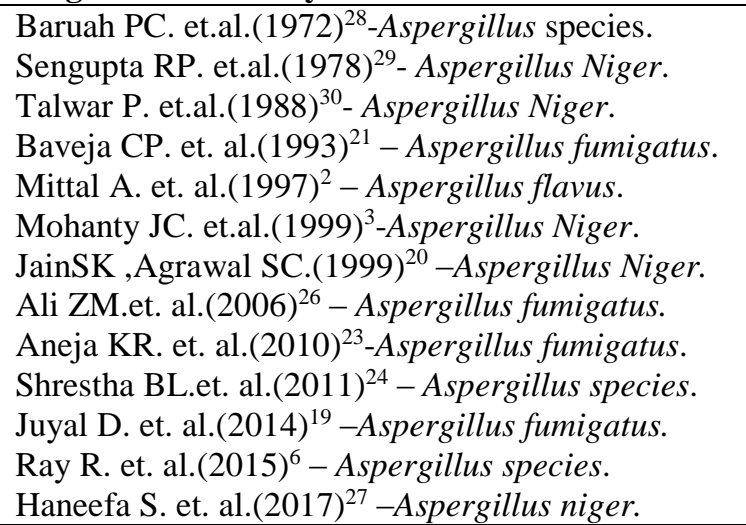 \\
\hline Candida & $\begin{array}{l}\text { Pasternale NI et.al.(1973) })^{31} \text {-Candida } \text { species, } \\
\text { Ballal M. et.al.(1992) })^{32}-\text { Candida albicans }\end{array}$ \\
\hline Penicillium & Baveja CP. et.al $(1993)^{21}$, Mohanty JC. et.al(1999) ${ }^{3}$ \\
\hline Cladosporium & Mittal A. et.al.(1997) ${ }^{2}$ \\
\hline Chrysosporium & Sampath CP. et.al( 2014) \\
\hline Mucor & Mohanty JC.et.al.(1999) ${ }^{3}$,Ray R. et. al.(2015) ${ }^{6}$ \\
\hline Other Saphrophytes & 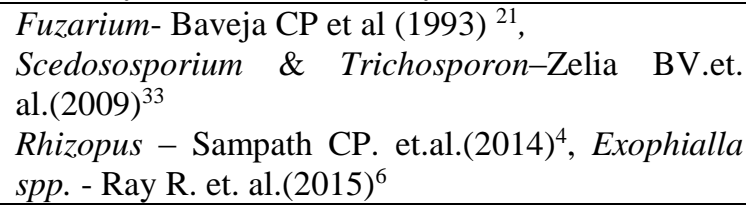 \\
\hline
\end{tabular}

\section{Conclusion}

In this study, we found that majority of the patients of ASOM and CSOM had clinico- mycological presentation similar to other parts of the world. Because of the variation in climate, community, patient population and inadvertent use of antibiotics, the pattern of microbiological and fungal pattern vary in both ASOM and CSOM. So it is very useful and helpful to find out these offenders of otitis media and so early diagnosis and treatment will possibly to help the patients.

\section{References}

1. Klein Jo. The burden of otitis media.Vaccine. 2000;19 (supple) 1:52-8.

2. Mittal A, Mann SBS, Naresh KP, MehraYN,Talwar P. Secondary fungal infections in chronic suppurative otitis media. Indian Journal of Otolaryngol and Head Neck Surgery 1997;2(49):112-6.

3. 3.Mohanty JC, Mohanty SK, Sahoo RC, Ghosh SK, Chayani N, Malliek B, et al. Clinico microbial profile of otomycosis in Berhampur. Indian Journal of Otology 1999;5(2):81-3.

4. Sampath CP, Subbannayya K, Manisha S, Nikhil DT. Primary Otomycosis in the Indian Subcontinent: Predisposing Factors, Microbiology, and Classification International Journal of Microbiology 2014, Article ID 636493, 9 pages.

5. Rippon JW. Medical Mycology: The Pathogenic Fungi and the Pathogenic Actinomycetes. $3^{\text {rd }}$ ed. Philadelphia: WB Saunders Company; 1988.

6. Ray R, Pal S, Ghosh M, Samaddar D and Banerjee M. Prevalence of Fungal infection in chronic otitis media-A study at a tertiary care hospital in Eastern India . International Journal of Current Microbiology and Applied Sciences 2015; 4(3):684-90. 
7. Deshmukh CT et.al. Acute otitis media in children treatment options. J Postgrad Med. 1998;44(3):81-4.

8. DVijaya, Geeta SH. Microbiological study of discharging otitis media. Indian Journal of Otology 2007;9 (3):17-19.

9. De A, Varaiya A, Tainwala S, Mathur M. Bacteriology of acute otitis media in children. Indian J Med Microbiol. 2002;20(1):54-5.

10. Kumar P. Bacteriology of acute otitis media. Indian Journal of Otology 2007; 13: 25-27.

11. Fule RP,Damude AS. Bacteriology of chronic suppurative otitis media .Indian Medical Gazette.1986; CXX (2):49-52.

12. Kulkarni RD, Dharmadhikari CA, Kulkarni VA, Udgaonkar US, Pawar SC.Bacteriological study of chronic otitis media. Indian Journal of Otolaryngology and Head Neck Surgery 1993; 2(2):110-3.

13. Sinha A, Kapil A, Gupta V. Aerobic bacteriological study of chronic suppurative otitis media. Indian Journal of otology 1999; 5(4):203-6.

14. Poorey VK, Iyer A. Study of bacterial flora in chronic suppurative otitis media and its clinical significance. Indian Journal of Otolaryngology and Head Neck Surgery 2002;54(2):91-4.

15. Taneja MK. Acute otitis media. Indian Journal of Otology 1998;4(4):161-4.

16. Gulati J, Tondon PL, Singh W, Bais AS. Study of bacterial flora in chronic suppurative otitis media.Indian Journal of Otolaryngology 1969;21:198-202.

17. Mawson SR and Ludman H: In:Diseases of the ear: A Textbook of Otology. Chicago Yearbook Medical Publishing; 1979, pp 328-30.

18. Kunelskaya et al. Fungal infect of post-operative of the ear.Vestn.Otorhinolaryngeal 1967;29:55-9.

19. Juyal D, Negi V, Sharma M, Adekhandi S, Prakash R, Sharma N. Significance of fungal flora in chronic suppurative otitis media. Ann Trop Med Public Health [serial online] 2014 [cited 2018 Mar 15];7:120-3

20. Jain SK,Agrawal SC.Sporostatic effects of some oils causing in otomycosis. Indian journal of medical sciences 1999;(46)1:1-6.

21. Baveja CP, Dhingra PL, Natrajan R. Mycological profile in otomycosis and its response to an antifungal agent. Indian Journal of Otolaryngology and Head Neck Surgery 1993;2(4):224-5.

22. Rajesh KY, Kumar G,Bansal M, Jaiswal A. International Journal of Contemporary Medical Research 2016 Aug;3(8).

23. Aneja KR, Sharma C, Joshi R. Fungal infection of the ear: A common problem in the north eastern part of Haryana. Int J PediatrOtorhinolaryngol 2010;74:604-7.

24. Shrestha BL, Amtya RCM, Shrestha I, Ghosh I. Microbiological profile of chronic suppurative otitis media .Nepalese Journal of ENT Head \& Neck Surgery 2011;2(2).

25. Dhingra R, MongaS, Kaur M, Sh, Brar RS, Rupali, andArora $\mathrm{H}$. Role of fungal infections in CSOM: Prospective study Indian Journal of Basic and Applied Medical Research; 2014 March; 3(2):598-608.

26. Ali ZM.Mycological studies in 15 cases of otomycosisPak J Med Sci 2006; 22(4):486-8.

27. Haneefa S,Bhama S and Rajahamsan J. Mycological Profile of Chronic Suppurative Otitis Media in a Tertiary Care Hospital inSouth India. Int.J.Curr.Microbiol.App.Sci2017;6(2):127-32.

28. Baruah PC, Agarwal SC, Arora MML. Clinical and microbiological studies in suppurative otitis in Chandigarh. Indian J of Otolaryngology.1972;24:157.
29. Sen Gupta RP, Kachker SK. Otomycosis. Indian J Med Science 1978;32:2-5.

30. Talwar P. Chakrabarti A, Poonamjit K. Fungal infection with special reference to chronic suppurative otitis media. Mycopathologica.1988;104:47-50.

31. Pasternale NI, Brysin VG and Danilova RD.Sensitization to mould and yeast like fungi in chronic purulent otitis media. Vestn.Otorhinolaryngol 1973;35:35-8.

32. Ballal M, Joyothirlatha, Kishor J, Rajan R , Shivananda PG. Chronic Suppurative otitis media: A bacteriological and mycological study. Indian Journal of Otolaryngology and HeadNeck Surgery 1992;1(1):11-2.

33. Zelia BV, Anna DFS, Edeltrudes DOL,MarcioDH ,Neuza MCO, Maria FF. et.al. Otomycosis: a retrospective study . Braz J Otorhinolaryngol 2009;75( 3):367-70. 